\title{
LA PRODUCCIÓN AGRARIA Y LA BIODIVERSIDAD EN LA NORMATIVA AGRARIA DE LA COMUNIDAD AUTÓNOMA DE LA RIOJA.
}

\author{
Dr. ANGEL SÁNCHEZ HERNÁNDEZ. \\ Profesor titular E.U. de Derecho civil \\ Universidad de La Rioja
}

SUMARIO

I. INTRODUCCIÓN. II. REGIMEN JURÍDICO DE LA UTILIZACIÓN DE MÉTODOS DE PRODUCCIÓN AGRARIA COMPATIBLES CON EL MEDIO AMBIENTE. III. NORMATIVA DE AMBITO ESTATAL. IV. NORMATIVA DE LA RIOJA. IV.1. Anexo I. Las "buenas prácticas agrarias habituales" que deben Respetarse: IV.2. Anexo II. Las ayudas para las siguientes actuaciones agroambientales: IV.2.1. Actuación primera: extensificación de la producción agraria. IV.2.2. Actuación segunda. Variedades autóctonas vegetales en riesgo de erosión genética. IV.2.3. Actuación tercera. Técnicas Ambientales de racionalización en el uso de productos químicos. - El control Integrado. - La Producción Integrada: Decreto 53/2001. - La agricultura ecológica: Decreto 56/2000. IV.2.4. Actuación cuarta. Lucha contra la erosión en medios frágiles. IV.2.5. Actuación quinta. Protección del paisaje y prácticas de prevención contra incendios. - Protección y mantenimiento del arbolado no productivo de la explotación. - Mantenimiento y conservación de cercas y muretes tradicionales, antiguos caminos agrícolas y pasos de ganado. -Conservación y mantenimiento de construcciones de chozos, lagares rupestres y construcciones asimiladas. IV.2.6. Actuación sexta. Gestión integrada de las explotaciones. - Mejora y conservación del medio físico. - Actuaciones sobre zonas de pastos y rastrojeras. Actuaciones sobre zonas de prados y pastizales. - Ganadería Ecológica. - Reducción de cabaña ganadera bovina, ovina y caprina por unidad de superficie forrajera. - Gestión racional de sistemas de pastoreo para la protección de flora y fauna. - Apicultura para mejora de la biodiversidad en zonas frágiles. 


\section{RESUMEN}

No cabe legislar en materia agraria sin considerar sus repercusiones en la flora y en la fauna, por lo que se hace preciso introducir nuevos instrumentos jurídicos para salvaguardar la biodiversividad. Esta necesidad se enmarca dentro de la Política Agrícola Común. La legislación de ámbito europeo constituye un marco coherente para las actuaciones de los Estados miembros y de sus entes regionales. En este marco comunitario, en España se aprueba, entre otros, el Real Decreto 4/2001 de 12 de enero por el que se establece un régimen de ayuda a la utilización de métodos de producción agraria compatibles con el medio ambiente. En la Rioja esta materia se regula en la Orden 6/2002 de 13 de febrero. El presente artículo analiza las medidas que podrán ser objeto de este tipo de ayudas con especial mención a la agricultura ecológica, la protección del paisaje y la gestión integrada de las explotaciones.

\section{INTRODUCCIÓN}

Las actividades agrarias necesarias para la sociedad, en los últimos siglos ha ido ocupando grandes extensiones de terreno y expulsando de ellas a la flora y fauna autóctonas o bien confinándolas en terrenos restringidos o inadecuados. Por otra parte, la introducción de métodos de producción agraria intensivos, ha dañado las poblaciones de animales y plantas silvestres, alterando un medio ambiente equilibrado para las especies silvestres que con idóneas prácticas agrícolas se había mantenido durante siglos.

Por tanto, se impone la conservación de los recursos naturales de la cual dependen factores económicos como el suelo de buena calidad y el agua no contaminada, elementos éstos que además de favorecer la conservación de la biodiversidad, constituyen un activo económico de garantía para las actividades agrícolas y ganaderas.

La conservación del medio ambiente es un problema al que no pueden hacer frente los Estados separadamente. Las aves migratorias no conocen fronteras, el caudal de los ríos transporta la polución en un viaje hacia el mar atravesando varios Estados y muchos de los hábitats, atraviesan las fronteras nacionales.

La Unión Europea y sus Estados miembros han contraído compromisos para la protección de la biodiversidad firmando convenios internaciones ${ }^{1}$, siendo el más importante el Convenio de Río de 1992, en el que establece el principio del desarrollo sostenible.

En la conferencia de las Naciones Unidas sobre Medio Ambiente y Desarrollo, celebrada en Río de Janeiro, durante el mes de junio de 1992, se firmó un convenio

\footnotetext{
${ }^{1}$ Me refiero a los siguientes convenios: Ramsar para la conservación de los humedales 1971, Helsinki sobre el mar Báltico 1974, Barcelona sobre el Mediterráneo 1976, Bonn sobre especies migratorias 1979, Berna para la protección de la fauna silvestre y los hábitats naturales 1979 y convenio para la protección de los Alpes 1991.
} 
sobre diversidad biológica. Además en esa llamada "cumbre de la Tierra", se aprobó la denominada "Agenda 21" que contempla un amplio plan con vistas a un desarrollo sostenible y a una protección ambiental global. El documento consta de cuarenta capítulos, de los cuales del noveno al vigésimo segundo, aluden al Medio Ambiente, con referencias entre otros a los siguientes aspectos: gestionar la tierra (el suelo) de forma sostenible (Cap. 10), combatir la deforestación (Cap. 11), combatir la desertización y la sequía (Cap. 12), desarrollo sostenible de áreas de montaña (Cap. 13), desarrollo agrícola y rural sostenible (Cap. 14) y conservación de la diversidad biológica, para lo cual se fomentarán métodos tradicionales de agricultura, agrosilvicultura, silvicultura, gestión de pastos que utilizan o mantienen o aumentan la biodiversidad $^{2}$.

Los Estados miembros de la Unión Europea han reaccionado para salvaguardar la biodiversidad en coexistencia con las actividades agrarias, creando nuevos instrumentos jurídicos que fomentan métodos tradicionales de producción agraria que han servido durante épocas para mantener una diversidad de habitats naturales y de la flora y fauna silvestres.

\section{REGIMEN JURÍDICO EN LA U. E. DE MÉTODOS DE PRODUCCIÓN AGRARIA COMPATIBLES CON EL MEDIO AMBIENTE .}

Las actuaciones a favor de la preservación de nuestro entorno natural cada vez adquieren más importancia en la normativa de la Unión Europea ${ }^{3}$. Ante los daños cada vez más importantes en la naturaleza y sus recursos, la protección de nuestro patrimonio natural - animales, plantas y paisajes característicos -, ha de integrarse en los objetivos de la normativa de la Unión Europea y de sus Estados miembros ${ }^{4}$. Se busca la coexistencia de la protección de la biodiversidad con determinadas actividades, que como la agricultura, la ganadería o la silvicultura, redundan en beneficio de la sociedad y de su progreso económico.

2 Sobre estos aspectos vide a KENTON R. MILLER, Instrumentos, estrategias y medidas internacionales para conservar la biodiversidad mundial, El Campo, $\mathrm{n}^{\circ}$ 128, abril-junio, 1993, p. 165 y ss.

${ }^{3}$ Entre la legislación más importante de la Unión Europea en materia de conservación de la naturaleza destacan la Directiva sobre aves, 79/409/CEE del Consejo, de 2 de abril de 1979, relativa a la conservación de las aves silvestres y la Directiva sobre hábitats 92/43/CEE del Consejo, de 21 de mayo de 1992, relativa a la conservación de los hábitats naturales y de la flora y fauna silvestres. Ésta última, con el fin de establecer una estrategia de desarrollo sostenible, identifica 200 tipos de hábitats, 200 especies de animales y más de 500 especies vegetales para su protección.

${ }^{4}$ Los Estados miembros de la Unión Europea ocupan una superficie de 3 millones de Kilómetros cuadrados y una población de 370 millones de habitantes. En este patrimonio natural se incluye una diversidad de formas de vida y ofrece una gran variedad de paisajes de una belleza impresionante. Se trata de miles de hábitats distintos en los que viven 150 especies de mamíferos, 520 especies de aves, 180 especies de reptiles y de anfibios, 150 especies de peces, 10.000 especies de plantas y al menos 100.000 especies de invertebrados- cifras de 1995 de la Agencia Europea del medio Ambiente (AEMA), Copenhague.

Sin embargo, muchas de estas especies están desapareciendo rápidamente. Actualmente, la mitad de nuestros mamíferos y un tercio de los reptiles, aves y peces están en peligro de estinción. Son 3000 las especies de plantas amenazadas, de las que 27 están en vías de extinción. 
No cabe, por tanto, legislar en materia agraria sin considerar sus repercusiones en la flora, en la fauna silvestre y en general en las zonas rurales. Se hace preciso introducir nuevos y firmes instrumentos jurídicos para salvaguardar una biodiversidad diversidad de los hábitats naturales y de la flora y fauna silvestres- que está en peligro.

Por otra parte, la protección de los hábitats naturales no significa reducir el desarrollo económico. Por el contrario, la protección del medio ambiente puede coexistir con los intereses sociales y económicos apostando por un desarrollo sostenible que mejore la calidad de vida, sin amenazar el bienestar de las futuras generaciones.

Dentro de las principales estrategias de la Unión Europea ${ }^{5}$, entre las que se encuentra la Política Agrícola Común, se tiene en cuenta la necesidad de conservar los paisajes y la flora y la fauna silvestres. Así, desde la agenda 2000 se ha recogido la protección del medio ambiente como uno de los principales objetivos de la reforma de la PAC, para alcanzar una producción agraria económica y ecológicamente viable ${ }^{6}$

La legislación de ámbito europeo constituye un marco coherente para las actuaciones de los Estados miembros y de sus entes regionales ${ }^{7}$. La legislación comunitaria en materia de medio ambiente respeta el principio de la subsidiariedad, esto es, deja en manos de las autoridades nacionales el establecimiento de las prioridades en este campo, reservándose el papel de apoyo y coordinación de las iniciativas, así como de comprobar que los Estados cumplen con sus obligaciones en la materia.

El Reglamento (CE) 1257/1999, del Consejo, de 17 de mayo, sobre ayudas al desarrollo rural, establece el marco de las ayudas comunitarias a favor de un desarrollo rural sostenible.

\section{NORMATIVA DE AMBITO ESTATAL.}

En este marco comunitario, en España se aprueba, entre otros, el Real Decreto $4 / 2001$, de 12 de enero, por el que se establece un régimen de ayudas a la utilización de métodos de producción agraria compatibles con el medio ambiente.

El reglamento (CE) 445/2002, de la Comisión, de 26 de febrero, establece disposiciones de aplicación del Reglamento 1257/1999, y origina en España la aprobación de un nuevo Real Decreto 708/2002, de 19 de julio, por el que se establecen medidas complementarias al Programa de Desarrollo Rural para las Medidas de Acompañamiento de la Política Agrícola Común. En el Capítulo III de este Real Decreto, se establecen medidas complementarias al Real Decreto 4/2001, de 12 de enero, por el que se establece un régimen de ayudas a la utilización de métodos de producción agraria compatibles con el medio ambiente. Concretamente se establecen como beneficiarios:

\footnotetext{
${ }^{5}$ El artículo 6 del Tratado de la Comunidad Europea establece que "las exigencias de la protección del medio ambiente deberán integrarse en la definición y en la realización de las políticas y acciones de la Comunidad ..., en particular con objeto de fomentar un desarrollo sostenible".

${ }^{6}$ Con la reforma de la PAC de 1992 se contribuyo a reducir el empleo de fertilizantes a base de nitrógeno y de fósforo.

${ }^{7}$ Ya en el Tratado de Maastricht de 1992 se afirmó que la Unión Europea incorpora la protección del medio ambiente a todas sus políticas.
} 
$1^{\mathrm{o}}$ - Los titulares de explotaciones agrarias que se comprometan, por un periodo mínimo de cinco años, salvo causa de fuerza mayor, a cumplir los compromisos de una o varias de las medidas agroambientales previstas en el anexo II del propio Decreto. Estas medias se refieren a la extensificación de la producción agraria, a las variedades autóctonas vegetales en riesgo de erosión genética, a las técnicas ambientales de racionalización en el uso de productos químicos- donde se alude a medidas referidas a la producción integrada y a la agricultura ecológica-, a la lucha contra la erosión en medios frágiles, a la protección de flora y fauna en humedales, sistemas especiales de explotación con alto interés medioambiental, al ahorro de agua de riego y fomento de la extensificación en la producción, a la protección del paisaje y prácticas de prevención contra incendios, y por último a gestión integrada de la explotaciones.

$2^{\circ}$ - Los agricultores que contraigan un compromiso agroambiental en relación con una parte de la explotación, deberán respetar en toda la explotación, como mínimo, las buenas prácticas agrarias habituales establecidas en el anexo I del propio Real Decreto. Se consideran como tales las técnicas normales de explotación que responsablemente aplicaría un agricultor en la zona donde ejerza su actividad, mencionándose las siguientes- si bien dada la diversidad de suelo agrario y clima de España, las Comunidades podrán establecer otras prácticas beneficiosas para el medio ambiente-: a) en relación con la conservación del suelo y lucha contra la erosión, se prohibe el laboreo convencional a favor de la pendiente; b) en cuanto a las alternativas y rotaciones, se consideran la existentes en las diferentes comarcas para conseguir una agricultura de desarrollo sostenible, aludiendo al barbecho en cualquiera de sus modalidades como una de las mejores prácticas agrícolas para los secanos españoles; c) optimización del uso de la energía fósil; d) utilización eficiente del agua; e) conservación de la biodiversidad - conservación de nidos de especies protegidas y prohibición de quemar los rastrojos y restos de cosecha, salvo que sea aconsejable su quema por motivos sanitarios o fitopatológicos previa autorización de la Comunidad Autónoma-; f) racionalización de uso de fertilizantes y prohibición de aplicar estiércoles y purines sobre terrenos encharcados o con nieve; g) Utilización racional de los herbicidas y productos fitosanitarios; h) eliminación de los materiales residuales utilizados en la producción y manejo adecuado de los restos de poda procedentes de los cultivos leñosos; i) no deben abandonarse los cultivos cuando se agote su capacidad productiva y, en cualquier caso, deberán mantenerse libres de plagas; j) debe cumplirse con lo establecido en materia de campañas oficiales de saneamiento ganadero con carácter obligatorio, k) no se debe sobrepasar la carga ganadera de las superficies forrajeras de la explotación que se establece en el Decreto y 1) en cualquier caso, además de aplicar las buenas prácticas agrícolas habituales anteriormente expuestas, se ha de respetar para ser beneficiario de las ayudas previstas, la legislación medioambiental referida a la conservación de los espacios naturales y de la flora y fauna silvestres $^{8}$, las medidas para contribuir a garantizar la biodiversidad mediante la conservación de hábitat naturales y de la flora y fauna silvestre ${ }^{9}$, las normas sobre protección de las aguas contra la contaminación producida por los nitratos procedentes de fuentes agrarias ${ }^{10}$ y la legislación de residuos ${ }^{11}$.

\footnotetext{
${ }^{8}$ Ley 4/1989, de 27 de marzo, modificada por las leyes 40/1997 y 41/1997.

${ }^{9}$ Directiva 92/43 (CE) y Real decreto 1997/1995, de 7 de diciembre.

${ }^{10}$ Directiva 91/679 (CE) y Real Decreto 261/1996, de 16 de febrero

${ }^{11}$ Ley 10/1998, de 21 de abril, de Residuos.
} 
$3^{\circ}$ - En el caso de la producción integrada, agricultura ecológica y ganadería ecológica, deben aplicarse respectivamente las medidas 3.2, 3.3. y 9.3 previstas en el Anexo II del Real Decreto 708/2002, en la totalidad de la superficie dedicada a la misma orientación productiva ubicada en cada explotación o, cuando ésta esté formada por varias fincas, en cada una de las fincas.

Como importante novedad el Real Decreto 708/2002, a los efectos del régimen de ayudas a la utilización de métodos de producción agraria compatibles con el medio ambiente, en su artículo 6, crea las Unidades Mínimas de Cultivo Agroambiental. Por tal se entiende la superficie agroambiental a partir de la cual los costes totales unitarios tienden a disminuir conforme crece el número de hectáreas cultivadas. Las Comunidades Autónomas fijarán el número de hectáreas por Unidad Mínima de Cultivo Agroambiental para cada medida agroambiental, sin que en ningún caso dicho numero de hectáreas pueda ser inferior a 20. Si las Comunidades Autónomas no establecen para alguna medida la superficie de la Unidad Mínima de Cultivo Agroambiental, ésta será igual a 20 hectáreas, a los efectos de calcular el importe de la prima.

El artículo 9 del Real Decreto 708/2002, establece en su Anexo III, establece una serie de medidas agroambientales incompatibles sobre una misma superficie, a los efectos de la percepción de primas, si bien las Comunidades Autónomas podrán ampliar la relación de medidas agroambientales incompatibles, cuando así lo aconsejen razones agronómicas o agroambientales o se pretenda evitar una duplicidad de primas por actuaciones análogas.

\section{NORMATIVA DE LA RIOJA.}

La Consejería de Agricultura, Ganadería y Desarrollo Rural, del Gobierno de La Rioja ha dictado la Orden 6/2002, de 13 de febrero, en la que se establecen las bases de un régimen de ayudas a la utilización de métodos de producción agraria compatibles con el medio ambiente

Este régimen de ayudas agroambientales tiene dos objetivos. En primero lugar, impulsar a los agricultores para que, "introduzcan o mantengan el uso de prácticas agrícolas que sean compatibles con la creciente necesidad de proteger y mejorar el medio ambiente, los recursos naturales, la diversidad genética y del suelo y de conservar el paisaje y el campo de la Comunidad Autónoma de La Rioja". En segundo lugar, alcanzar los objetivos comunitarios en materia de agricultura y medio ambiente y corregir los problemas de carácter agroambiental con los que se enfrentan las explotaciones agrarias españolas y riojanas.

La Orden establece las normas de aplicación, en la Comunidad Autónoma de La Rioja, del Real Decreto 4/2001 de 12 de enero, por el que se establece un régimen de ayudas a la utilización de métodos de producción agraria compatibles con el medio ambiente - recientemente complementado por el Real Decreto 708/2002, de 19 de julio(Artículo 1).

Podrán ser objeto de ayuda las actuaciones agroambientales que están comprendidas en las medidas siguientes (Artículo 2 ): 
1.- Extensificación de la producción agraria.

2.- Variedades autóctonas en riesgos de erosión genética.

3.- Técnicas ambientales de racionalización en el uso de productos químicos.

4.- Lucha contra la erosión en medios frágiles.

5.- Protección del paisaje y prácticas de prevención contra incendios.

6.- Gestión integrada de las explotaciones ganaderas extensivas.

A los efectos de esta Orden, la Unidad Mínima de Cultivo Agroambiental (UMCA) -referencia para determinar la superficie agroambiental a partir de la cual los costes totales unitarios tienden a disminuir conforme crece el numero de hectáreas cultivadas-, en la Comunidad Autónoma de La Rioja para las medidas y submedidas será de 20 hectáreas, excepto en el caso de la apicultura para mejora de biodiversidad en zonas frágiles, que será de 150 hectáreas (Artículo 9).

\section{IV.1. Anexo I. Las "buenas prácticas agrarias habituales" que deben respetarse:}

1. Se conservará el suelo como recurso natural básico y se luchará contra la erosión.

2. Se optimizará la utilización de la energía utilizada en la maquinaria agrícola.

3. Se Utilizará eficientemente el agua.

4. Se conservará la diversidad biológica referida a :

a) Material vegetal, cumpliendo todos los requisitos exigidos por la normativa vigente sobre la producción, comercialización y utilización de semillas y plantas de vivero.

b) La conservación de los nidos de especies protegidas de difícil localización, tomándose las medidas adecuadas para evitar daños en las tareas de recolección.

c) La quema de rastrojos o pastos de cosecha queda prohibida. En el caso de que sea aconsejable proceder a su quema por motivos sanitarios o fitopatológicos, el beneficiario deberá disponer de la correspondiente autorización de la Consejería de Agricultura, Ganadería y Desarrollo Rural.

d) Las zonas con posibles riesgos de incendio, ya sea porque limiten con lugares en los que se puedan originar incendios (caminos muy transitados, líneas férreas, lugares habitados, etc.) o porque haya en las mismas restos de cosecha muy combustibles o espacios de alto valor paisajístico, se aislarán mediante franjas labradas de, al menos, tres metros de anchura.

5. Se racionalizará el uso de fertilizantes y los estiércoles y purines no se aplicarán sobre terrenos encharcados o con nieve y cuando las explotaciones se encuentren en las zonas vulnerables, se evitará estercoleros estancos. 
6. Se realizará una utilización racional de los productos fitosanitarios y herbicidas, dentro de los márgenes establecidos por la normativa, la gestión de envases se hará conforme a las normas establecidas.

7. Se reducirá la contaminación de origen agrario, eliminando los materiales utilizados en la producción y los restos de poda, cuya permanencia sobre el terreno, por su incidencia sobre la proliferación de plagas y enfermedades, no se considera conveniente.

Los restos derivados de los plásticos usados y otros residuos deberán retirarse de las parcelas y depositarse en lugares apropiados.

Se manejarán adecuadamente los restos de poda procedentes de los cultivos leñosos tales, como la vid, olivo, frutales de secano y de regadío y árboles con aprovechamiento ganadero. Es práctica tradicional el consumo de las partes verdes por el ganado, la obtención de leña de los restos de diámetro adecuado y el apilado y quema del resto. Esta forma de actuar es una buena práctica agraria que debe conservarse, ya que los aportes de $\mathrm{CO} 2$ a la atmósfera son mínimos y, en cambio, los beneficios por lo que se refiere al control de plagas y enfermedades de los cultivos son importantes.

8. Los cultivos finalizados, una vez agotada su vida útil económica, no se deberán abandonarse y, en cualquier caso, deberán mantenerse libres de plagas, enfermedades, parásitos susceptibles de ser transmitidos a otras propiedades, con riesgos de pérdidas económicas o agroambientales.

En cuanto a la Sanidad animal, las explotaciones que se acojan a un programa agroambiental ganadero deberán llevar un programa sanitario preventivo, especialmente en todo lo relacionado con el control de parásitos, externos e internos y las enfermedades de declaración obligatoria, cumplir con lo establecido en materia de Campañas Oficiales de Saneamiento Ganadero y con todo lo establecido por la normativa vigente en materia de uso de alimentos prohibidos y de anabolizantes.

\section{IV.2. Anexo II ${ }^{12}$, se contemplan las ayudas para las siguientes actuaciones agroambientales:}

IV.2.1. Actuación primera: extensificación de la producción agraria, encaminada a la protección del suelo, así como incrementar su contenido en materia orgánica y su actividad microbiana. Con ello se consigue importantes mejoras en su capacidad de retención de agua y una menor necesidad de fertilización mineral. Para ello se:

$1^{\circ}$ - mejorará el barbecho tradicional y se mantendrá en buenas condiciones.

\footnotetext{
${ }^{12}$ En el Anexo III, se contienen las principales incompatibilidades entre medidas agroambientales; en el Anexo IV, las definiciones aclaratorias y en el Anexo V se hace referencia de las dosis de fertilizantes recomendados para aplicación de la medida 3 .
} 
$2^{\circ}$ - establecerán sistemas de extensificación para la protección de la flora y la fauna. Se pretende la preservación de determinadas especies animales -aves-y vegetales de interés ambiental. Para ello, se realizarán las siguientes actuaciones ambientales:

-Utilizar semillas que no contengan productos fitosanitarios que pongan en peligro la supervivencia de la avifauna esteparia.

- Enterrado del rastrojo anterior del 15 de abril.

-Mantener durante el periodo de duración de los compromisos linderos e islas de vegetación espontánea, en al menos, un 3\% de la explotación, en forma de fajas lineales o bosquetes.

- Respetar el calendario de prácticas agrícolas (recolección y alzado de los rastrojos) que fijará el Comité Técnico en función de los periodos de nidificación.

- Incremento en la dosis de semilla en $20 \mathrm{Kg} / \mathrm{ha}$. para compensar las pérdidas producidas por las aves.

$3^{\circ}$-Retirada de tierras de la producción para la creación de espacios reservados para la fauna y conservación de la biodiversidad

Con esta medida se pretende la recuperación de entornos agrarios de alto valor paisajístico, favorables al mantenimiento de la biodiversidad, recuperando su riqueza florística y faunística.

Para ello se llevarán se realizarán, en una superficie de, al menos, 1 hectárea las actuaciones de mantenimiento necesarios para evitar la erosión, riesgo de incendios y deterioro de la cubierta vegetal; podas de arbolado, desbroces mecánicos del material arbustivo que supere el $30 \%$ de la superficie, etc., quedando Prohibido el uso de agroquímicos.

\section{IV.2.2. Actuación segunda. Variedades autóctonas vegetales en riesgo de erosión} genética.

Pretende el mantenimiento de la biodiversidad y riqueza genética.

Consiste en fomentar la recuperación de determinadas variedades de especies vegetales en peligro de extinción, aludiendo a cultivos específicos, mantenimiento superficies en franca regresión, acompañándose de prácticas agroambientales tales como la racionalización de productos químicos, para así contribuir doblemente, a la conservación de los recursos y a la protección y mantenimiento de la diversidad genética.

La superficie mínima acogida será de 0,3 hectáreas y las especies autorizadas son variedades autóctonas vegetales en riesgo de erosión genética como :

- Caparrón de Anguiano.

- Caparrón pinto de Castañares.

- Garbanzo de Pedroso. 
- Pimiento Najerano.

- Pimiento de Santo Domingo.

- Aneas.

- Nogal de Pedroso.

IV.2.3. Actuación tercera. Técnicas Ambientales de racionalización en el uso de productos químicos.

Se pretende introducir prácticas de cultivo que, con la reducción en el uso de productos químicos, disminuyan los efectos contaminantes sobre las aguas, suelo y la atmósfera, reduciendo la toxicidad en los productos.

"La creación de mecanismos voluntarios a los cuales se pueden acoger los agricultores, tales como la difusión de mejoras en prácticas agrícolas, puede llevar a reducciones muy apreciables de la contaminación medioambiental. Sin embargo la adopción de dichas prácticas agrícolas por parte de los agricultores conlleva una reducción del margen bruto. Los agricultores adoptarán una determinada tecnología siempre que no les produzca pérdidas económicas, por lo que es necesario al menos compensar mediante ayudas a aquellos agricultores que se comprometan a adoptar mejoras prácticas medioambientales".

Para ello, durante cinco años en la superficie acogida, se mantendrán setos y ribazos, vegetación en lindes y márgenes para reserva ecológica y mantenimiento de la biodiversidad y se reducirá, al menos un $20 \%$ de los productos agroquímicos utilizados en la explotación, se establecerá un plan de fertilización y el control de malas hierbas se realizará de forma mecánica o mediante pastoreo controlado. Dentro de esta actuación se distinguen tres medidas distintas:

a) El control Integrado que trata de reducir la potencial contaminación de suelos y aguas, fomentando la adopción de métodos de lucha natural, biológica o tecnológica contra los organismos nocivos, es decir, se sustituirá el tratamiento químico por lucha biológica.

Para ello durante un periodo, al menos de cinco años, se reducirán, al menos, un $25 \%$ de los tratamientos químicos y se realiza un control biológico en, al menos, el $50 \%$ de la superficie y siempre por parcelas completas, aplicando preferentemente métodos de lucha natural o biotecnológica en el resto de las parcelas. Se realizará el seguimiento y control de plagas, mediante tratamientos dirigidos y localizados para preservar la fauna útil.

\section{b) La Producción Integrada}

Dirigida a la reducción de efectos contaminantes de aguas y suelos, fomentando la adopción de metodologías de producción vegetal que utilizan al máximo los recursos y los mecanismos de producción naturales y asegurando a largo plazo una agricultura sostenible y la protección de los recursos naturales. 
Para ello las actuaciones agroambientales se dirigirán a la reducción de los tratamientos químicos al menos en un $30 \%$, teniendo prioridad de los métodos biológicos, físicos o culturales frente a los químicos en la lucha contra plagas y se mantendrá la cubierta vegetal en cultivos perennes. En épocas de recolección se permitirá la siega o el pastoreo controlado para facilitar las labores de cosecha. En viñedo se mantendrá, al menos, en los meses de otoño e invierno. No podrá realizarse escarda química.

\section{Concepto de producción integrada.}

La producción integrada es un sistema de producción que pretende alcanzar una agricultura de calidad y sostenible. Dos son por tanto los objetivos:

En primer lugar, potenciar una agricultura e calidad que se consolide como una agricultura de futuro. Se trata de obtener alimentos y otros productos de elevada calidad utilizando recursos naturales y mecanismos reguladores que reemplazan los insumisos contaminantes.

La producción integrada, no sólo alcanza a la producción, sino también a la transformación y comercialización de alimentos y otros productos agrarios.

En segundo lugar, se busca un equilibrio necesario entre agricultura y medio ambiente para alcanzar una agricultura situada entre la agricultura convencional y la ecológica, que asegure una producción agraria sostenible, dando preferencia a métodos y técnicas más respetuosos con el entorno, minimizando la utilización de productos químicos que generen efectos secundarios sobre el medio ambiente y la salud humana. En suma, se trata de realizar un uso sostenible de los recursos, con su máximo aprovechamiento y la disminución de productos externos, con lo que se reducen los costes de producción y se incrementa la protección del medio ambiente.

\section{Regulación de la producción integrada.}

En la Comunidad Autónoma de La Rioja la producción integrada está regulada mediante el Decreto 53/2001, de 21 de diciembre.

El Decreto define la producción integrada como "el sistema de producción agraria, transformación y comercialización que utiliza al máximo los recursos y los mecanismos de producción naturales" ${ }^{13}$. Regula el objeto y ámbito de aplicación: a) Se establecen las normas de producción y los requisitos generales que deben cumplir quienes se acojan al sistema de producción, transformación y comercialización de los productos agrarios; b) Se regula, el uso de la marca de garantía de producción integrada de La Rioja, la vigencia del control de la misma, el registro de productores acogidos al sistema de Producción integrada y el registro de elaboradores de producción integrada; y por último c) se reconoce la figura de la agrupación de producción integrada (APRIAS) ${ }^{14}$.

\footnotetext{
${ }^{13}$ Artículo 1 del Decreto 53/2001, de 21 de diciembre.

${ }^{14}$ Artículo 1 del Decreto 53/2001, de 21 de diciembre.
} 
En cuanto a las competencias, será la Consejería de Agricultura, Ganadería y Desarrollo Rural, a través del Instituto de Calidad Agroalimentaria, quien ejercerá las funciones de control de las normas técnicas, de la promoción y defensa de la marca de garantía de producción integrada ${ }^{15}$.

Respecto a las normas de producción, los productores que pretendan que sus productos sean comercializados bajo distintivo de la marca de garantía de producción integrada deberán reunir los siguientes requisitos: a) estar inscritos en el registro de productores, b) cumplir las normas de producción, c) someter su explotación al régimen de controles e instrucciones específicas, d)pertenecer a una "agrupación de producción integrada" o tener un contrato con un Ingeniero para la asistencia o dirección técnica, e) disponer de un cuaderno de explotación en el que se reflejen las operaciones culturales y sanitarias, f) comprometerse a que el personal técnico que desarrolle tareas de producción integrada asista a los cursos que al efecto se programen, g) obtener la totalidad de la producción de una parcela agrícola por el sistema de producción integrada, estando aquella claramente separada de otras que no estén sometidas a las normas del presente Decreto, h)garantizar que durante la manipulación y el transporte no puede haber sustitución de los productos, i) notificar anualmente su programa de producción y por último, j) hacer buen uso de la marca de garantía de producción integrada ${ }^{16}$.

El Decreto regula igualmente las agrupaciones de producción integrada ${ }^{17}$, el registro de productores ${ }^{18}$, certificado de productos de producción integrada ${ }^{19}$. En las normas de elaboración se definen las fases de almacenamiento, conservación, transformación, elaboración, etiquetado y venta de productos acogidos al sistema de producción integrada. Se señala los requisitos que debe reunir un elaborador que pretenda que sus productos sean comercializados con el distintivo de la marca de garantía de producción integrada ${ }^{20}$. Se contempla un registro de elaboradores de producción integrada ${ }^{21}$, un certificado de elaborador de producción integrada ${ }^{22}$, la marca de garantía de producción integrada, con su correspondiente logotipo, cuya titularidad corresponde a la Comunidad Autónoma de La Rioja ${ }^{23}$. Se regula la utilización de la marca de garantía ${ }^{24}$, por los productores o elaboradores de producción integrada con una vigencia de cinco años ${ }^{25}$, si bien cabe la revocación y la suspensión de la autorización del uso de la marca de garantía ${ }^{26}$. Las entidades de control serán el Instituto de Calidad Agroalimentaria o bien las entidades privadas reconocidas por Resolución de

\footnotetext{
${ }^{15}$ Artículo 2 del Decreto 53/2001, de 21 de diciembre.

${ }^{16}$ Artículo 3 del Decreto 53/2001, de 21 de diciembre.

${ }^{17}$ Artículo 4 del Decreto 53/2001, de 21 de diciembre.

${ }^{18}$ Artículo 5 del Decreto 53/2001, de 21 de diciembre.

${ }^{19}$ Artículo 6 del Decreto 53/2001, de 21 de diciembre.

${ }^{20}$ Artículo 7 del Decreto 53/2001, de 21 de diciembre.

${ }^{21}$ Artículo 8 del Decreto 53/2001, de 21 de diciembre.

${ }^{22}$ Artículo 9 del Decreto 53/2001, de 21 de diciembre.

${ }^{23}$ Artículo 10 del Decreto 53/2001, de 21 de diciembre.

${ }^{24}$ Artículo 11 del Decreto 53/2001, de 21 de diciembre.

${ }^{25}$ Artículo 12 del Decreto 53/2001, de 21 de diciembre.

${ }^{26}$ Artículo 13 del Decreto 53/2001, de 21 de diciembre.
} 
la Consejería de Agricultura ${ }^{27}$, en base a los requisitos mínimos de control establecidos $^{28}$.

El Decreto 53/2001, de 21 de diciembre, está desarrollado por cuatro Ordenes de la Consejería de Agricultura, Ganadería y Desarrollo Rural: Orden 1/2002, de 13 de febrero, por la que se dispone la publicación del Reglamento de uso de la marca garantía "Producción integrada de La Rioja" y de su distintivo ${ }^{29}$; Orden 2/2002, de 13 de febrero, por la que se establece el procedimiento de inscripción en los registros de productores y elaboradores de agricultura de producción integrada de la Comunidad Autónoma de La Rioja ${ }^{30}$; Orden 3/2002, de 13 de febrero, por la que se establece el procedimiento para la concesión de autorizaciones para la utilización de la marca de garantía "Producción integrada de La Rioja" ${ }^{31}$; Orden 4/2002, de 13 de febrero, por la que se regula el sistema de control y certificación de la producción integrada en la Comunidad Autónoma de La Rioja ${ }^{32}$.

\section{Prácticas agrícolas que se deben cumplir en la producción integrada.}

El Decreto 53/2001, en su Anexo I, establece las normas generales de producción integrada, definiendo "las prácticas agrícolas que, bajo la dirección del responsable del servicio técnico competente, deben cumplir los elaboradores con independencia de la legislación vigente, especialmente en cuanto a material vegetal, fertilizantes, fitosanidad, gestión de residuos y envases, prevención de riesgos laborales, sanidad y medio ambiente." 33

${ }^{27}$ Artículo 14 del Decreto 53/2001, de 21 de diciembre.

${ }^{28}$ Artículo 15 del Decreto 53/2001, de 21 de diciembre.

${ }^{29}$ B.O.R. $n^{\text {o. }} 21$ del 16 de febrero de 2002.

${ }^{30}$ B.O.R. no .21 del 16 de febrero de 2002.

${ }^{31}$ B.O.R. no .21 del 16 de febrero de 2002.

${ }^{32}$ B.O.R. n'. 21 del 16 de febrero de 2002.

${ }^{33}$ Se establecen, entre otras, las siguientes normas técnicas en relación a las prácticas agrícolas mencionadas seguidamente:

$1^{\circ}$ - En la preparación del terreno, se respetará al máximo la estructura del suelo, evitando las escorrentías, y los encharcamientos. Los sustratos inertes deben ser reciclados adecuadamente. Queda prohibido: la desinfección del suelo mediante tratamientos químicos, salvo excepciones; el uso de PVC en los materiales de acolchado y el abandono de plásticos, envases y otros residuos en el medio ambiente.

$2^{\circ}$ - En la siembra o plantación, hay que emplear material vegetal procedente de productores autorizados y utilizar semillas debidamente desinfectadas, quedando prohibidos determinados patrones y combinaciones injerto-patrón o variedades especialmente sensibles a determinadas enfermedades de especial incidencia o relevancia.

$3^{\circ}$ - En las enmiendas y fertilización es obligatorio el suministro de nutrientes vía suelo así como la fertilización mineral adecuada a todos los parámetros de cultivo. Queda prohibido superar la cantidad máxima

$4^{\circ}$ - En el laboreo, es obligatorio, en los cultivos cubiertos, arranque de malas hierbas, así como la retirada de plásticos para su reciclado y en las épocas adecuadas.

Las prácticas de conservación del suelo se realizarán en función de la pendiente. La cubierta vegetal espontánea se mantendrá mientras no afecte a la producción. Cuando sea imprescindible utilizar herbicidas, se usará la técnica de herbigación o aplicación localizada, siempre con materias activas autorizadas.

Queda prohibida la utilización de aperos que destruyan la estructura del suelo y propicien la formación de suelo de labor. 


\section{Ventajas de la producción integrada.}

En suma, la producción integrada beneficia :

a)-a los consumidores, por obtener alimentos con más calidad y seguridad.

b)-a los agricultores, ganaderos y silvicultores que con menos coste, con obtendrán mejores precios por sus productos, incrementando así sus rentas evitándose así el éxodo rural.

c)-al medio ambiente, pues al utilizar métodos de producción menos agresivos, se respeta la flora, la fauna, la biodiversidad, se reduce la erosión, se racionaliza el uso del agua y se reduce la contaminación de las aguas subterráneas.

\section{c) La agricultura ecológica.}

La agricultura ecológica tiene cada vez más importancia en España, como lo pone de manifiesto la evolución en los últimos años que ha experimentado un considerable aumento, tanto en el número de productores como en el número de hectáreas dedicadas a este sistema de producción. Este tipo de agricultura esta regulada en el Reglamento (CE) $n^{\circ}$ 2092/91 del Consejo de 24 de junio de agricultura ecológica o biológica ${ }^{34}$, así como el Real Decreto 1852/93 de 22 de octubre sobre la producción agrícola ecológica del M.A.P.A. y el Decreto 56/2000 de la Consejería de Agricultura, Ganadería y Desarrollo Rural de la C.A. de La Rioja.

La agricultura ecológica establece métodos de cultivo compatibles con el medio ambiente a través de un catálogo de conductas agroambientales muy exigentes. Además

$5^{\circ}$ - La poda, si es de cultivos leñosos, debe respetarse el estado fisiológico óptimo de la planta, quedando prohibida la quema incontrolada de resto de poda y el abandono de los restos de oda en la parcela.

$6^{\circ}$ - En el riego, se dispondrá de las características analíticas de la calidad del agua de riego, estableciéndose su volumen en función de la profundidad reticular, las características del suelo y valores de conductibilidad intolerables. Deben utilizarse métodos de riego que garanticen la mayor eficiencia en el uso del agua.

Queda prohibida la utilización de aguas residuales sin autorización y el empleo de aguas no acordes con los parámetros de calidad intolerables para el cultivo.

$7^{\circ}$ - En el control de plagas y enfermedades, es obligado anteponer métodos biológicos, físicos o genéticos a los métodos químicos. Cuando sea imprescindible una intervención química, las materias activas a utilizar deben estar autorizadas, de acuerdo con criterios de menor impacto ambiental, toxicidad, problemas de residuos y efectos sobre la fauna auxiliar. Las malas hierbas se controlarán, siempre que sea posible, con medios mecánicos.

Queda prohibida en los cultivos hortícolas, la utilización de herbicidas dentro del invernadero, así como de herbicidas remanentes en suelos arenosos.

También queda prohibido el abandono de envases de productos fitosanitarios en el medio ambiente...

${ }^{34}$ Téngase presente que el anexo III del Reglamento (CEE) no 2092/91,del Consejo sobre producción agrícola ecológica y su indicación en los productos agrarios y alimentarios, ha sido sustituido por el anexo del Reglamento (CE), no 2491/2001 de la Comisión de 19 de diciembre de 2001, referido a los "requisitos mínimos de control y medidas precautorias establecidos dentro del régimen de control contemplado en los artículos 8 y $9 "$ ". 
del cumplimiento de las normas de producción ecológica, no se emplearán abonos de síntesis química y productos químicos para el control de plagas y enfermedades.

\section{Significado de la agricultura ecológica.}

La agricultura ecológica entraña replantearse el modo de producción agraria: cultivar las tierras y criar el ganado de modo orgánico, sin ayuda de elementos químicos de síntesis.

La producción agraria ecológica tiene como objetivo primordial, además de buscar productos agropecuarios sanos elaborados con métodos que mantienen la integridad y las cualidades vitales del producto, la protección y preservación del medio ambiente, bien manteniendo la fertilidad del suelo a largo plazo, o reduciendo al mínimo el empleo de recursos no renovables, o bien, por último, reduciendo al mínimo la contaminación del agua, del suelo y aire derivados de prácticas agrícolas. Para ello resulta esencial, además del vínculo entre la agricultura y la naturaleza, el respeto de los equilibrios naturales, que mantienen a la agricultura lejos de la intervención y del empleo de productos de síntesis.

En suma, la agricultura ecológica " es un sistema global de producción agrícola vegetales y animales- que prefiere el empleo de métodos de cultivo biológicos y mecánicos al de productos químicos sintéticos ${ }^{35}$.

\section{Reglamento CEE $n^{\circ} 2092 / 91$ del Consejo, sobre producción agrícola ecológica y su indicación en los productos agrarios y alimentarios ${ }^{36}$.}

Inicialmente este Reglamento únicamente fijó normas para la producción ecológica de origen vegetal. En 1995, se contemplan dichas normas con la posibilidad de crear un logotipo para la agricultura ecológica y otras normas técnicas en relación con el etiquetado y el régimen de importación. En 1999 se aprueba el Reglamento CE 1084/1999, de 19 de julio, en el que se fijan las normas comunitarias relativas a la producción ecológica de origen animal. En marzo de 2000 se creó un logotipo comunitario para la agricultura ecológica. Por último, el anexo III del Reglamento (CEE) nº 2092/91, fue sustituido por el anexo del Reglamento (CE), nº 2491/2001 de la Comisión de 19 de diciembre de 2001, referido a los "requisitos mínimos de control y medidas precautorias establecidos dentro del régimen de control contemplado en los artículos 8 y $9 "$.

\footnotetext{
${ }^{35}$ Vide directrices para la producción, elaboración, etiquetado y comercialización de alimentos producidos orgánicamente, aprobadas en junio de 1999 por la Comisión Codex alimentarius, de un programa mixto FAO/OMS, sobre los alimentos, al objeto de garantizar la seguridad alimentaria y lealtad de las transacciones comerciales en este ámbito.

${ }^{36}$ Fue completado por Reglamento CEE 1084/1999 de 24 de agosto de 1999, y modificado por el anexo III del Reglamento (CEE) no 2092/91,del Consejo sobre producción agrícola ecológica y su indicación en los productos agrarios y alimentarios, ha sido sustituido por el anexo del Reglamento (CE), $n^{\circ}$ 2491/2001 de la Comisión de 19 de diciembre de 2001, referido a los "requisitos mínimos de control y medidas precautorias establecidos dentro del régimen de control contemplado en los artículos 8 y 9 ".
} 
El Reglamento CEE 2092/91 determina los requisitos que debe cumplir un producto agrícola o un alimento para llevar algún tipo de referencia al método de producción ecológica; define en qué consiste el método de obtención de productos vegetales y animales ecológicos: regula el etiquetado y la transformación, la inspección y el comercio de estros productos ecológicos en la Unión Europea así como la importación de productos ecológicos de terceros países.

\section{Ámbito de aplicación del Reglamento CEE $n^{\circ} 2092 / 91$.}

Se aplica a los productos vegetales y animales no transformados, a los productos agrícolas transformados destinados a la alimentación humana y a los piensos para animales, siempre que lleven en el etiquetado indicaciones para sugerir al consumidor que el producto se ha obtenido mediante el método de producción ecológica ${ }^{37}$. A los productos agrícolas ecológicos le son de aplicación las disposiciones generales por las que se regulan la producción, la elaboración, la comercialización, el etiquetado y el control de los productos agrícolas y convencionales ${ }^{38}$.

\section{Normas relativas a la producción ecológica.}

\section{Vegetal.}

La fertilidad y la actividad biológica del suelo deben mantenerse o incrementarse, siguiendo un programa de rotación anual ${ }^{39}$. Esta medida puede completarse incorporando a la tierra estiércol procedente de explotaciones ganaderas ecológicas. Cuando estas medidas sean insuficientes para lugar una nutrición adecuada de los vegetales o para acondicionar la tierra y sea necesario incorporar fertilizantes orgánicos o minerales, pero únicamente los consistentes básicamente en minerales naturales poco solubles que no se obtienen mediante síntesis química.

Se evitarán al máximo la utilización de productos fitosanitarios a fin de proteger a las plantas contra los parásitos, las enfermedades y la eliminación de las malas hierbas.

La protección de los vegetales pasa, en primer lugar por una selección de especies y de variedades que sean resistentes por naturaleza; en segundo lugar, pasa por la aplicación de programas de rotación de cultivos y en último lugar por la protección de los enemigos naturales de los parásitos (conservación de setos, nidos, etc...) ${ }^{40}$.

\footnotetext{
${ }^{37}$ Artículo 1 del Reglamento CEE n ${ }^{\circ}$ 2092/91 del Consejo, de 24 de junio de 1991, sobre la producción ecológica y su indicación en los productos agrarios y alimentarios, DOCE L 198 de 22 de julio de 1991.

${ }^{38}$ Así ocurre con todas las normas sobre seguridad de los productos para la salud humana, artículo 2 del Reglamento CEE n⿳ 2092/91 del Consejo, de 24 de junio de 1991, sobre la producción ecológica y su indicación en los productos agrarios y alimentarios , DOCE L 198 de 22 de julio de 1991.

${ }^{39}$ Punto 2, parte A del anexo I del Reglamento CEE n ${ }^{\circ}$ 2092/91.

${ }^{40}$ Punto 3, parte A del anexo I del Reglamento CEE n ${ }^{\circ}$ 2092/91. Caso de que un peligro inmediato amenace al cultivo, al normativa autoriza a que se utilicen productos fitosanitarios que figuren en la lista de la parte B del anexo II del Reglamento, vg. productos a base de microorganismos.
} 
El periodo mínimo para convertir una parcela de agricultura convencional en una agricultura ecológica es de dos años, antes de la siembra, en el caso de cultivos anuales; $\mathrm{y}$ de tres años, antes de la primera cosecha, en el de los cultivos vivaces distintos de las praderas. Estos periodos pueden prorrogarse o reducirse en función de la utilización anterior de las parcelas ${ }^{41}$.

En cuanto a los vegetales que crecen espontáneamente en zonas naturales y bosques, su recolección se asimila a un método de producción ecológica siempre que cumplan dos requisitos. En primer lugar, durante los tres años anteriores, dichas zonas no se hayan tratado con productos prohibidos en la producción ecológica. En segundo lugar, la recolección no debe afectar a la estabilidad del hábitat natural ni a la supervivencia de las especies de la zona ${ }^{42}$.

\section{Animal.}

La cría ecológica de animales se orienta en el principio de un fuerte vínculo entre los animales y las fincas. Este necesario vinculo con la tierra obliga a que los animales tengan acceso a zonas de ejercicio al aire libre y asimismo a que reciban una alimentación no sólo ecológica sino preferentemente producida en la propia granja.

Los principios generales aplicables a la producción ecológica animal son básicamente dos ${ }^{43}$. El primero el principio de complementariedad entre el suelo y los animales - producción ligada al suelo- que supone la exclusión de la producción en establos de tipo cerrado - los animales deben disponer de animales al aire libre -, siendo la densidad animal por hectárea limitada. En segundo lugar se menciona el principio de separación, consistente en que todos los animales criados en una misma unidad de producción deben criarse cumpliendo las normas de la producción ecológica. Excepcionalmente es posible una producción ecológica y convencional si existen garantías suficientes que permitan evitar la confusión entre producción ecológica convencional.

Se contemplan unas normas sobre el periodo de conversión y sobre el origen de los animales. Unas se refieren a la constitución de un rebaño, seleccionando las razas que mejor se adopten al entorno y sean más resistentes a las enfermedades. Los animales procederán de una explotación que respete las normas de la agricultura ecológica y criarse posteriormente según esas normas a lo largo de toda su vida. Otras normas se refieren a la alimentación de los animales, realizándose con productos ecológicos que procedan preferentemente de la propia explotación -vg. mamíferos alimentados con leche natural-. Por último, se establecen también unos principios aplicables a la profilaxis y a los cuidados veterinarios: se da prioridad a la prevención, basada en la selección de razas apropiadas; aplicación de prácticas zootécnicas que refuercen la resistencia de animales; mantenimiento de una densidad de animales adecuada. Si estas medidas resultan insuficientes y surge una enfermedad se dará preferencia a los

\footnotetext{
${ }^{41}$ Punto 1 de la parte A del Anexo I del Reglamento CEE no 2092/91.

${ }^{42}$ Punto 4 de la parte A del Anexo I del Reglamento CEE no 2092/91

${ }^{43}$ Véase el punto primero de la parte B del Anexo I del Reglamento CEE no 2092/91, modificado el 19 de julio de 1999 por el Reglamento CE n 1804/99, que establece normas mínimas para la producción ecológica animal.
} 
tratamientos naturales sobre los que tienen antibióticos que pueden dejar residuos en los productos. Los tratamientos con antibióticos se utilizarán si resultan imprescindibles para curar al animal. esta prohibido el uso de sustancias destinadas a estimular el crecimiento -vg. hormonas- o a controlar la reproducción ${ }^{44}$.

Normas relativas a la transformación de los productos agrícolas ecológicos en alimentos.

Se limita, sin excluirlos totalmente, los ingredientes de origen no agrario -aditivos aromatizantes, agua, sal preparados a base de microorganismos-, así como los auxiliares tecnológicos que resultan imprescindibles para elaborar alimentos a partir de productos agrícolas de origen ecológico ${ }^{45}$.

Se prohibe emplear organismos modificados genéticamente y tratamientos ionizantes, así como utilizar al mismo tiempo un ingrediente producido ecológicamente y el mismo ingrediente pero producido de manera convencional ${ }^{46}$.

\section{Normas relativas al etiquetado y a la publicidad.}

El etiquetado y la publicidad sólo pueden hacer referencia al método de producción ecológica si las indicaciones dejan claro que se trata de un método de producción agraria y el producto cumple las normas del Reglamento CEE 2092/91.

Ahora bien, sólo cabe hacer referencia al método de producción ecológica cuando al menos el 95\% de los ingredientes han sido obtenidos por ese método. Esto es, hasta un $5 \%$ pueden ser ingredientes producidos de forma convencional, siempre que esos productos no se encuentren en el mercado comunitario de productos ecológicos o se encuentren en cantidades insuficientes.

Los productos que tengan un porcentaje de ingredientes ecológicos de entre el $70 \mathrm{y}$ el 95\% sólo pueden hacer referencia a este método de producción en la lista de ingredientes no en la denominación de venta. Si fuesen menos del 70\% los ingredientes de un producto que son de origen agrícola ecológico, no se puede hacer ninguna referencia al método de producción ecológica en el etiquetado o en la publicidad del producto.

\section{El logotipo de los productos agrícolas ecológicos.}

\footnotetext{
${ }^{44}$ En el punto 6.1. de la parte B del anexo I del Reglamento CE $n^{\circ} 1804 / 99$ se establecen normas del bienestar del animal. Prácticas como el corte del rabo o el descuerne están sujetas a autorización que procederá únicamente por motivos de seguridad, higiene, salud o bienestar de los animales. Está prohibido mantener atados a los animales y las condiciones de los locales de cría y alojamiento deben responder a las necesidades fisiológicas y etológicas de los animales. El transporte de los animales debe realizarse siempre respetando el bienestar animal y de tal forma que el estrés sea mínimo.

${ }^{45}$ Parte B del Anexo VI del Reglamento CEE no 2092/91.

${ }^{46}$ Vide el artículo 5 del Reglamento 2092/91.
} 
En el Reglamento (CEE) no 2092/91, según la modificación efectuada en 1995 Reglamento (CE) 1935/95 del Consejo, de 22 de junio de 1995- se ofrecía a la Comisión Europea la posibilidad de aprobar un logotipo específico para la producción ecológica.

En mayo de 2000, la Comisión aprobó el logotipo específico para la producción ecológica por medio del Reglamento (CE) no 331/2000 con el objetivo por una parte de dar mayor credibilidad a los productos ecológicos entre los consumidores, y por otra parte con el fin de mejorar su identificación en el mercado.

Este logotipo sin ser obligatorio, sólo lo puede llevar los productos regulados por el Reglamento (CEE) $n^{\circ} 2092 / 91$ que cumplan cuatro condiciones ${ }^{47}$. La primera, tener al menos un $95 \%$ de ingredientes producidos según las normas de la agricultura ecológica. La segunda, haber estado sometidos al régimen de control previsto por el Reglamento durante todo el proceso de producción y de elaboración. La tercera, venderse directamente en envases sellados o comercializarse como alimentos preenvasados -en envases o contenedores- para hacer imposible sustituir su contenido; la cuarta y última es llevar en la etiqueta el nombre y/o la razón social de productor, elaborador o vendedor así como el número de código del organismo de certificación.

\section{Medidas de Control.}

La producción ecológica debe someterse al sistema específico de control establecido por el Estado miembro, que en todo caso ha de observar los requisitos mínimos de control y medidas precautorias establecidas dentro del régimen de control previstos en el Anexo III del Reglamento (CE) no 2491/2001 de la Comisión de 19 de diciembre de 2001.

\section{La producción ecológica en la Comunidad Autónoma de La Rioja ${ }^{48}$.}

Según el Reglamento (CEE) 2092/91, el control de la producción agraria ecológica debe ser realizado por autoridades de control a designar por los Estados miembros. En el caso de España -como expresamente reconoce el artículo 5 del R. D. 1852/1993, de 22 de octubre, sobre producción agrícola ecológica y su indicación en los productos agrarios y alimenticios ${ }^{49}$, por los órganos competentes de las Comunidades

\footnotetext{
${ }^{47}$ Vide el artículo 10 del Reglamento CEE no 2092/91 y Reglamento CE no 331/2000 de la Comisión, de 17 de diciembre de 1999 - por el que se modifica el anexo V del Reglamento (CEE) n 2092/91, DOL 48 de 19 de febrero de 2000.

${ }^{48}$ La agricultura y ganadería ecológica de La Rioja en cifras

Las cifras de la agricultura ecológica en la Comunidad Autónoma de La Rioja a finales del año 2001 son las siguientes: Superficie inscrita, 2374 hectáreas; Tipos de productos, en hectáreas: frutales, 130, frutos secos 600 , olivo 155 , vid 240 , hortalizas 80 , pastizales 445 , silvícola 300 , semillas y viveros 0,4 , apicultura 1917, ganado caprino 185, anual extensivo 420. Las industrias son 46: de vinos 17, de aguardientes de orujos 3, vinagrerías 2, frutas y hortalizas frescas 3, frutos secos 1 , aceite de oliva 3, miel 6 , conservas vegetales 6 , panadería y pastelería 2 , medicinales aromáticas 1 , quesería 1 , compost 1.

${ }^{49}$ Recientemente por Real Decreto 506/2001, de 11 de mayo se ha modificado el Real Decreto 1852/1993, de 22 de octubre, sobre producción agrícola ecológica y su indicación en los productos
} 
Autónomas. En este sentido, desde 1996 las Competencias de control y certificación fueron transferidas a la Comunidad Autónoma de La Rioja.

Para determinar la autoridad competente y estructurar la autoridad de control sobre esta materia dentro del ámbito de la Comunidad Autónoma de La Rioja se dicta Decreto $56 / 2000$, de 17 de noviembre, sobre producción agrícola ecológica e indicación de la misma en los productos agrarios y alimentarios y se crea el Consejo de la Producción Agraria Ecológica de La Rioja.

El Decreto 56/2000, de 17 de noviembre, de la Consejería de Agricultura, Ganadería y Desarrollo Rural, sobre producción agrícola ecológica e indicación de la misma en los productos agrarios y alimentarios y se crea el Consejo de Producción Agraria Ecológica de la Rioja, regula en primer lugar la producción, la elaboración y la comercialización de productos ecológicos, esto es, productos agrícolas vegetales no transformados, productos animales y productos animales sin transformar en la medida en que se adecuen a lo dispuesto en los anexos I y III del Reglamento CEE 2091/91; productos agrícolas vegetales transformados y productos animales transformados destinados a la alimentación humana, preparados básicamente a partir de uno o más ingredientes de origen vegetal o animal; y por último, alimentos para animales, piensos compuestos y materias primas para la alimentación animal no recogidos en el párrafo a) en las condiciones establecidas ${ }^{50}$ por el Reglamento (CE) 1804/1999.

En segundo lugar el Decreto 56/2000, se ocupa de los instrumentos de fermento, promoción, control y asesoramiento en materia de actividad agraria ecológica. Los productos ecológicos se distinguirán del resto con la aparición en las etiquetas, la publicidad o en los alimentos animales de las palabras ecológico, biológico y "orgánico" o de los prefijos "bio" y "eco" 51 . Para no inducir a confusión al consumidor, se prohibe la utilización de estas denominaciones en los alimentos que no hayan sido producidos con métodos ecológicos ${ }^{52}$.

Las funciones de la autoridad de control, "órgano de control de la Agricultura Ecológica de La Rioja", se ejercerá por la Dirección General de Desarrollo Rural ${ }^{53}$. Se crea el Consejo de la producción agraria ecológica de La Rioja, para ejercer las funciones de consulta y colaboración en materia de agricultura ecológica ${ }^{54}$. Da normas técnicas de producción y elaboración ${ }^{55}$ y se establecen las obligaciones de los operadores $^{56}$ de notificar la actividad a la Consejería, someterse a los controles necesarios y cumplir las normas técnicas referentes a la naturaleza de los productos, de

agrarios y alimenticios, BOE de 26 de mayo de $2001, \mathrm{n}^{\circ} 126$.

${ }^{50}$ Vide el artículo único punto 1 del RD 506/2001, de 11 de mayo por el que se modifica el RD 1852/1993, de 22 de octubre sobre producción agrícola ecológica y su indicación en los productos agrarios y alimentarios. Vide también el artículo 1 del Decreto 56/2000, de 17 de noviembre, de La Rioja.

${ }^{51}$ Vide el artículo 2.1 del Decreto 56/2000 de La Rioja.

${ }^{52}$ Vide el artículo 2.2 del Decreto 56/2000 de La Rioja.

${ }^{53}$ Vide el artículo 4 del Decreto 56/2000 de La Rioja.

${ }^{54}$ Vide el artículo 5 del Decreto 56/2000 de La Rioja; en su artículo 12 describe sus competencias, en el artículo 13 sus funciones, en el artículo 14 su composición, en el 15 el régimen de las sesiones y en el artículo 16 se ocupa del presidente del consejo.

${ }^{55}$ Vide el artículos 6 y 7 del Decreto 56/2000 de La Rioja.

${ }^{56}$ Vide el artículos 8 y 10 del Decreto 56/2000 de La Rioja. 
las fincas, métodos de producción y elaboración previsto, al uso de determinados fertilizantes, agentes antiparasitarios y fármacos.

Se crean los registros de operadores ${ }^{57}$. Se establece un sistema de control ${ }^{58}$.Los controles se realizarán de forma periódica y para ello los operadores de agricultura ecológica facilitarán las facturas y justificantes necesarios, las muestras que se requieran $\mathrm{y}$ el acceso a las instalaciones.

En cuanto a la indicación de conformidad ${ }^{59}$. Los productos agrarios y alimentarios ecológicos llevarán una contra-etiqueta numerada con el logotipo y en la que figuran las inscripciones "La Rioja" y "ES-RI-AE", Organo de control de la Agricultura Ecológica" y además del número y la serie.

\section{IV.2.5. Actuación cuarta. Lucha contra la erosión en medios frágiles.}

Existen comarcas en la Comunidad Autónoma de La Rioja, que por el predominio del clima árido o semiárido, se encuentran en una situación de elevado riesgo de erosión.

Las características combinadas de nuestra climatología y orografía, así como la acción del hombre que con sus actuaciones sobre el terreno favorece la acción de los agentes naturales, son los responsables de los procesos de erosión.

"Esta medida va especialmente dirigida al mantenimiento de cultivos adecuados a zonas de pendiente y a la introducción de sistemas de mínimo laboreo que permitan el mantenimiento de una cubierta vegetal para su utilización por la fauna autóctona".

Se luchará contra la erosión en medios frágiles:

-En cultivos leñosos- olivar, almendro- en pendiente superior al $8 \%$ o en terrazas y bancales, manteniendo y conservando los elementos e instalaciones tradicionales relacionadas con el cultivo: muretes, terrazas, bancales, setas vegetales, etc. que quedarán reflejadas en el plan de actuación agroambiental. En todo caso, queda prohibido el uso de productos químicos para la poda y eliminación de brotes.

-Y en el mantenimiento de tierras abandonadas, evitando el riesgo de pérdidas de suelo y de incendios que, de otra forma, se podrían producir en superficies agrícolas abandonadas. Las actuaciones agroambientales se dirigen a la protección de la cubierta y realización de podas y limpiezas en zonas agrarias marginales abandonadas al cultivo en los tres últimos años al menos.

IV.2.6. Actuación quinta. Protección del paisaje y prácticas de prevención contra incendios.

\footnotetext{
${ }^{57}$ Vide el artículo 9 del Decreto 56/2000 de La Rioja.

${ }^{58}$ Vide el artículo 10 del Decreto 56/2000 de La Rioja.

${ }^{59}$ Vide el artículo 11 del Decreto 56/2000 de La Rioja.
} 
"Esta medida va dirigida a la conservación del paisaje, de árboles, limpieza de vegetación a fin minimizar los riesgos de incendios". Las acciones de esta medida se dirigen a la protección del paisaje: mantenimiento de elementos de singular valor paisajístico de la explotación.

Su objetivo "es favorecer la integración de la actividad agraria con su entorno natural, bien a través de conservar el paisaje como elementos del ecosistema, bien a través de la conservación de elementos específicos". Para ello se desarrollaran las siguientes actuaciones agroambientales:

- Se conservará la cubierta vegetal, los setos y bosquetes, mediante el control del crecimiento de la vegetación con un pastoreo controlado, se eliminará la maleza de los pies de los árboles y capa arbustiva con medios mecánicos y se enterrara y/o recogerá o se elaborará compost con restos de poda, residuos agrícolas y restos de alimentos del ganado.

- Se mantendrá durante 5 años la actividad agraria en la parcela donde se encuentre ubicado el elemento objeto de la ayuda.

\section{$1^{\circ}$ - Protección y mantenimiento del arbolado no productivo de la explotación.}

Esta medida pretende conservar durante 5 años los elementos paisajísticos de la explotación mediante:

-podas de árboles y eliminación de ramas muertas y enfermas y reposición de pies enfermos y muertos y

- la recogida de restos de poda y ramas caídas.

- la conservación de la vegetación natural, los árboles o arbustos aislados y las isletas de zarzas o matorral arbustivo.

$2^{\circ}$ - Mantenimiento y conservación de cercas y muretes tradicionales, antiguos caminos agrícolas y pasos de ganado

Esta medida pretende el mantenimiento y reconstrucción de cercas y muros de piedra tradicionales que serán revisadas y reparadas anualmente, así como el mantenimiento de antiguos caminos agrícolas y pasos de ganado, mediante limpieza de la vegetación de las mismas y mantenimiento de las cunetas.

$3^{\circ}$ - Conservación y mantenimiento de construcciones de chozos, lagares rupestres y construcciones asimiladas

La medida pretende la reparación y conservación de las obras de fábrica de chozos, lagares rupestres y otras construcciones asimiladas.. y la reparación y sustitución periódica de la cubierta . 
Será necesario que el número medio de chozos o lagares rupestres sea de una para cada 5 hectáreas en el conjunto de la explotación o en la parte de la misma acogida a esta medida.

Las características de las construcciones son :

"Chozos: Estas construcciones, que parecen surgir de la misma tierra, forman parte del paisaje de la Sonsierra y están íntimamente ligadas a la labor primordial de la comarca.

También reciben el nombre de "guardaviñas" por ser el lugar desde el que se vigilaban los viñedos evitando los robos. Además, presentan la función de proteger tanto a los viñadores como a los jornaleros de las inclemencias del tiempo durante las labores anuales, dado el alejamiento de las tierras del domicilio habitual.

Su tipología es muy variada, presentando las siguientes variaciones:

Cuevas excavadas en la arcilla

Cuevas excavadas en la arcilla con frente de piedra

Cuevas excavadas en la roca

Refugios que aprovechan salientes de la roca, excavados y con una pared de piedra para protección del aire.

Casetas de planta cuadrada o rectangular con cubierta a dos aguas.

Chozos de planta cuadrada o circular de falsa cúpula y semicirculares.

Chozos de planta circular de falsa cúpula y cónicos.

Lagares rupestres: Estas construcciones se hallan totalmente excavadas en la roca y están formadas por un gran recinto circular en desnivel, lugar donde se depositaba el fruto de la vid, y un deposito adosado de una profundidad superior, comunicado con el anterior por medio de un pequeño canalillo. En este depósito se recogía el jugo de la uva, más fácil de transportar en la antigüedad.

Construcciones asimiladas: otras construcciones tradicionales de las zonas de pastoreo y cultivo."

\section{IV.2.7. Actuación sexta. Gestión integrada de las explotaciones}

La aplicación de esta medida va encaminada a la realización de una gestión racional de la explotación compatibilizando la conservación del medio físico con las labores de la gestión ganadera. Ase contemplan diversas actuaciones agroambientales:

\section{$1^{o}$ - Mejora y conservación del medio físico}

Se pretende conservar ecosistemas agrarios y sus recursos, haciendo una gestión racional de los aprovechamientos ganaderos y actuaciones de protección de la cubierta. 


\subsection{Actuaciones sobre zonas de pastos y rastrojeras}

Con esta medida requiere las siguientes practicas agroambientales:

- un respeto a las cargas ganaderas máximas y mínimas establecidas, en superficies mínimas de actuación de 15 hectáreas.

- un aprovechamiento racional de las rastrojeras y resto de superficies pastables, respetando el calendario de pastoreo.

- no levantar los rastrojos hasta 5 meses después de la recolección de las superficies de cereales integrantes de su explotación agrícola que figuren en la declaración anual de cultivos herbáceos.

- dejar al menos el 50\% de la paja de los cultivos de cereales sobre el terreno.

- recoger las cuerdas del atado de pacas de paja, heno o ensilado utilizadas para alimentación del ganado, almacenándolas en la explotación en sacos hasta su retirada.

\subsection{Actuaciones sobre zonas de prados y pastizales.}

Esta medida requiere de las siguientes actuaciones agroambientales y requisitos :

- Una superficie mínima de actuación de 7 hectáreas en las explotaciones con aprovechamientos de montes comunales y de 3 hectáreas en las restantes.

- Mantener el aprovechamiento extensivo de las superficies afectadas, respetando el calendario de aprovechamientos.

- Comprometerse a mantener al menos el 75\% incluidos los aprovechamientos comunales, de la superficie agraria útil (S.A.U.) de la explotación de pastos o cultivos orientados a la alimentación de volumen del ganado.

- Conservación y mantenimiento de elementos ganaderos necesarios para un adecuado manejo de los pastos (evitar sobre y subpastoreo).

- $\quad$ Respeto a las cargas ganaderas anuales máximas y mínimas

\section{$2^{\circ}$.Ganadería Ecológica}

"Se fomenta la protección de los recursos naturales, agua, suelo y paisaje, y la reducción de los potenciales focos y niveles de contaminación a través de la acogida de una metodología de gestión sostenible de la explotación, como es la producción ganadera ecológica sobre superficies acogidas a la agricultura ecológica" Respeto a cargas ganaderas máxima y mínimas establecidas.

Esta medida requiere el cumplimiento de los requisitos establecidos para acogida a la producción vegetal ecológica descritos en el Reglamento 2092/91 de Producción 
Ecológica y Reglamento Comunitario $1804 / 99$ de 24 de agosto, sobre producción ganadera ecológica.

En cuanto a la apicultura ecológica, se establecen las siguientes actuaciones agroambientales:

- Mantener más de 50 colmenas.

- Localizar las colmenas en superficies cultivadas o pastizales ecológicos y no en superficies no cultivadas o que no estén en agricultura ecológica.

- No utilizar alimentos compuestos que contengan polen.

- Existirá una densidad máxima de una colmena por ha.

- Las colmenas se localizarán en superficies cultivadas ecológicamente y/o pastizales ecológicos.

\section{$3^{\circ}$. Reducción de cabaña ganadera bovina, ovina y caprina por unidad de superficie forrajera.}

"Su objetivo es la protección del suelo, disminuyendo de forma efectiva la carga ganadera en praderas y pastizales que han sufrido un desequilibrio por el pastoreo excesivo en su composición florística, con la subsiguiente aparición de especies no deseables para el ganado, fomentando sistemas de aprovechamiento de pastos racionales, adaptados a cargas ganaderas que permitan explotaciones ganaderas más extensivas con una reducción efectiva de, al menos, el 20\% de la inicial”.

\section{$4^{o}$. Gestión racional de sistemas de pastoreo para la protección de flora y fauna.}

"La medida está dirigida a la conservación de la biodiversidad y a la protección de la flora y fauna. Propone fomentar una gestión racional de pastos con la reducción efectiva de la carga ganadera de la explotación de origen, ausentándose de la misma, al menos, un periodo de 4 meses, con desplazamiento físico del ganado, contribuyendo a mejorar los aprovechamientos, consiguiendo la recuperación del suelo y de su diversidad florística, tanto de la explotación de origen como la receptora".

Se realizarán las siguientes actuaciones agroambientales:

- Realizar la práctica de la gestión tradicional de pastos con desplazamiento estacional del ganado con el mismo número de animales durante al menos 5 años consecutivos.

- Realizar el movimiento del ganado de acuerdo a las prácticas tradicionales de aprovechamiento de forma óptima de los recursos.

- Permanecer fuera de la explotación de origen al menos cuatro meses, a contar desde junio. Durante estos cuatro meses es obligatorio cambiar de pastos, desplazando los animales a pastizales de montaña con aprovechamiento estacional. 
- Reducir la carga ganadera al menos en un $25 \%$ en la explotación de origen, durante el periodo que dura el aprovechamiento de pastos fuera de la explotación.

\section{$5^{\circ}$. Apicultura para mejora de la biodiversidad en zonas frágiles.}

La medida se desarrollará en una superficie de vegetación entomófila, caracterizada por ecosistemas con biodiversidad frágil propios de praderas y pastizales, así como todas las zonas con biodiversidad frágil y vegetación autóctona, con una colmena cada 2 hectáreas de vegetación autóctona. Cada colmenar no tendrá mas de 80 colmenas y la distancia entre ellos será superior a $1 \mathrm{Km}$., debiendo ubicarse permanentemente en zonas de biodiversidad frágil; excepto durante los seis meses de trashumancia que pueden realizar en $80 \%$ de las colmenas como máximo. No se suministrará alimentación estimulante que contenga polen. 